\title{
Prognostic factors in pulmonary arterial hypertension: assessing the course of the disease
}

\author{
L.S. Howard
}

ABSTRACT: The practical management of pulmonary arterial hypertension (PAH) requires an accurate assessment of disease severity and prognosis. A number of prognostic indicators are known to be associated with patient outcome, and recent treatment guidelines advocate using such parameters to guide management decisions. Although $\mathrm{PAH}$ is characterised by the presence of pulmonary vasculopathy, it is the response of the right ventricle to an increased afterload that is the greatest determinant of a patient's symptoms and survival; thus, measurements that capture right ventricular function provide the best potential to assess PAH severity. One challenge is to understand how the tests we use in everyday clinical practice relate to right heart function in PAH patients, and how current measures can be improved and developed to optimise assessment of disease status and progress. Future research in the field of PAH should focus on how best to assess right heart function, and which measures or combination of measures provide the most relevant information for the individual patient.

\section{KEYWORDS: Haemodynamics, pulmonary arterial hypertension, right heart failure}

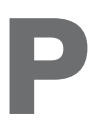
ulmonary arterial hypertension $(\mathrm{PAH})$ is a serious and progressive disease of the pulmonary vasculature that can be triggered by a number of known (genetic mutations, toxins, infections or other diseases) and unknown causes. The pathogenesis of PAH is multifactorial, involving a number of biochemical pathways and cell types [1], but all forms of $\mathrm{PAH}$, regardless of underlying aetiology, share a common pulmonary vasculopathy characterised by intimal proliferation, medial hypertrophy and the development of plexiform lesions. Together with associated vasoconstriction and the development of in situ thrombosis, this proliferative and obstructive remodelling of pulmonary blood vessel walls leads to a progressive increase in pulmonary vascular resistance (PVR) and, subsequently, increased pulmonary arterial pressure $(\mathrm{Ppa})$ with a resultant increase in afterload on the right ventricle. The ability of the right ventricle to adapt to this afterload is the key determinant of symptoms and survival, and ultimately, although characterised by pulmonary vasculopathy, it is the failure of the right ventricle that is the main cause of death in patients with PAH [2]. In view of this, measures of cardiac function and not just the extent of the pulmonary vascular disease take on particular significance when assessing central cardiopulmonary haemodynamics. Factors reflecting right ventricular function as assessed by right heart catheterisation (RHC), including mean right atrial pressure (Pra), cardiac output and cardiac index, have been shown to be significant predictors of survival in $\mathrm{PAH}$ [3-5]. In addition, a range of other factors that reflect right ventricular structure and function, including echocardiographic parameters, exercise capacity and serum biomarkers, have been shown to be of prognostic value. In order to identify the best way to use such surrogate markers in assessing the course of disease in PAH, we need to understand not only how the primary lesion, i.e. pulmonary vascular remodelling, is triggered and develops, but also how the right ventricle adapts and how its function deteriorates over time. This article will discuss how disease progression of $\mathrm{PAH}$ is currently assessed in clinical practice, and explore the use of multifaceted follow-up using a range of markers of right heart function and structure.

\section{CHANGES IN CARDIAC FUNCTION DURING THE COURSE OF PAH}

In the pre-symptomatic stage of $\mathrm{PAH}$, it is thought that increases in PVR and resting mean $P$ pa do not overtly affect resting cardiac function,
CORRESPONDENCE

L.S. Howard

National Pulmonary Hypertension

Service - London

Dept of Cardiac Sciences

Hammersmith Hospital

Imperial College Healthcare NHS

Trust

Du Cane Road

London

W12 OHS

UK

E-mail: I.howard@imperial.ac.uk

Received:

Aug 312011

Accepted after revision:

Sept 192011

PROVENANCE

Publication of this peer-reviewed article was supported by Actelion Pharmaceuticals Ltd, Switzerland (principal sponsor, European Respiratory Review issue 122). 
such as cardiac output (fig. 1). By the time a patient presents with symptoms, even with "early" symptoms, classified as being in World Health Organization (WHO) functional class (FC) II, PVR is already substantially above normal, suggesting advanced pulmonary vascular remodelling. In the EARLY (Endothelin Antagonist Trial in Mildly Symptomatic Pulmonary Arterial Hypertension Patients) trial, $\mathrm{PAH}$ patients classified as WHO FC II had a mean PVR at entry of 822 dyn $\cdot \mathrm{cm} \cdot \mathrm{s}^{-5}$ [6], approximately 10 -fold higher than healthy individuals. Although the right ventricle initially compensates for this increase in afterload through adaptive hypertrophy and remodelling, this process is not entirely benign and cannot be maintained as overload is sustained over time; eventually the right ventricle dilates and fails [7]. Cardiac dysfunction manifests with both diastolic and systolic impairment and, crudely, this can be measured at catheterisation as an increase in Pra and fall in cardiac output.

While PVR describes the fundamental haemodynamic abnormality underlying $\mathrm{PAH}$, it does not follow that it is a key indicator of prognosis. This is true, even more so, for mean $P$ pa, for which a fall may either indicate a fall in PVR or cardiac output. Right ventricular mass and size, and Pra reflect functional status and are strong predictors of survival $[8,9]$.

The challenge, therefore, is to understand how the assessments available for use in current clinical practice reflect the function of the right ventricle in PAH. These assessments can be considered as two separate categories: those directly measuring cardiac function, for example stroke volume or measures of right ventricular contractility or filling pressure; and those that are integrative measures of right ventricular failure, such as exercise capacity, neuroendocrine activation or biomarkers. It is important to bear in mind that no single parameter can be relied upon alone to predict prognosis, and current treatment guidelines recommend that the evaluation of disease severity and response to therapy should be based on the assessment of a number of parameters with proven prognostic significance that reflect symptoms and exercise capacity (table 1) [9]. These

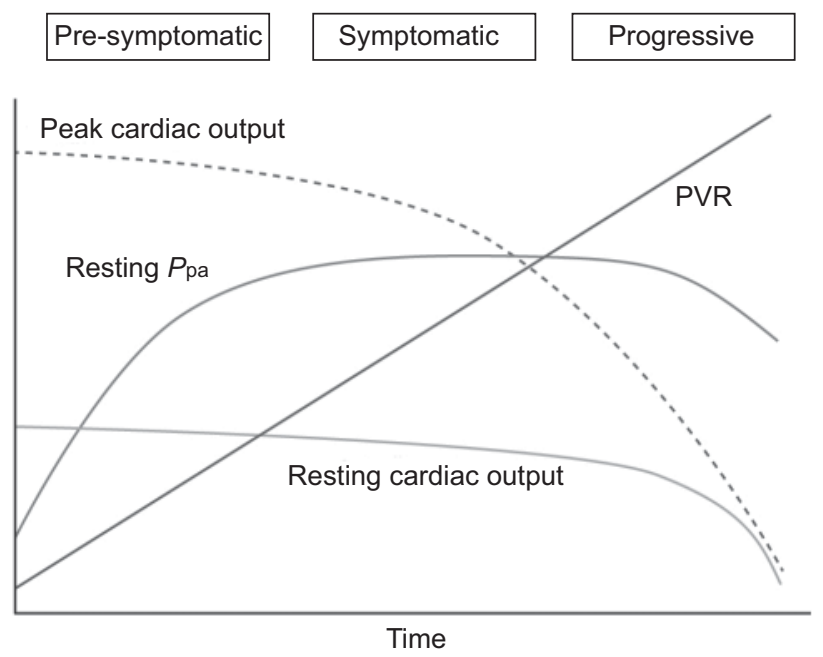

FIGURE 1. Schematic representation of changes in haemodynamic parameters over the course of pulmonary arterial hypertension. Ppa: pulmonary arterial pressure; PVR: pulmonary vascular resistance. parameters and the way in which they reflect right ventricular failure in PAH are discussed below.

\section{INDIVIDUAL MEASURES USED IN THE ASSESSMENT OF PAH PATIENTS \\ Clinical evidence of right heart failure}

A number of clinical symptoms or signs, such as peripheral oedema and the onset or increasing severity of angina, can be signs of deteriorating right ventricle function. In particular, patients who begin to experience syncope or who experience an increase in the frequency of syncopal episodes have poor prognosis and require immediate attention [9]. Recently, syncope has been shown to be an independent predictor of mortality after adjusting for multiple factors [10].

\section{Functional class}

The WHO functional classification contextualises a patient's symptoms (dyspnoea, fatigue, chest pain, syncope) in relation to their everyday activity. Clinical evidence of right heart failure, rate of progression of symptoms and the presence of syncope are all considered as prognostic indicators in PAH (table 1), and all three of these symptoms are encapsulated within the WHO functional classification. WHO FC itself is a strong predictor of survival, both before and during treatment [4, 11]. As such, WHO FC is a particularly useful and effective tool for determining the severity of PAH and frequent assessment of WHO FC is a key recommendation in recent guidelines and consensus documents $[9,12]$.

Despite WHO FC being a good all-round indicator of disease severity, it is a subjective measure and is open to over- or underestimation arising from differences in the perception of symptom impact by both patient and clinician. It may also be confounded by comorbidities such as obesity and age, and by coexisting or underlying diseases; for example, patients with systemic sclerosis (SSc)-associated PAH may have their symptoms and exercise capacity limited by musculoskeletal factors [13]. In addition, lower WHO FC does not necessarily equate to less severe haemodynamic impairment and some patients may present in WHO FC II with minimal symptoms while also displaying a high degree of haemodynamic compromise; such patients are likely to deteriorate rapidly $[6,14]$. Although undoubtedly valuable, WHO FC alone is insufficient to determine prognosis in all patients, and further surrogate markers are required.

\section{Exercise testing}

Exercise testing, either in the form of a walking test, such as measurement of 6-min walk distance (6MWD), or via cardiopulmonary exercise testing (CPET), can provide a global measurement of right heart function, since there is a direct relationship between maximal cardiac output and oxygen delivery and consumption. Both assessments are relatively easy to perform regularly and form an important part of the recommended follow-up procedure for PAH patients [9].

6MWD is currently the only Food and Drug Administration and European Agency for the Evaluation of Medicinal Products accepted exercise end-point for studies evaluating treatment effects in PAH in adults [9]. It is a good indicator of prognosis [4] and has been shown to decrease in proportion to the severity of WHO FC, and to correlate with cardiac output, total pulmonary 


\begin{tabular}{|c|c|c|}
\hline Slow & Rate of progression of symptoms & Rapid \\
\hline No & Syncope & Yes \\
\hline I, II & WHO FC & IV \\
\hline Peak $\mathrm{O}_{2}$ consumption $>15 \mathrm{~mL} \cdot \mathrm{min}^{-1} \cdot \mathrm{kg}^{-1}$ & Cardio-pulmonary exercise testing & Peak $\mathrm{O}_{2}$ consumption $<12 \mathrm{~mL} \cdot \mathrm{min}^{-1} \cdot \mathrm{kg}^{-1}$ \\
\hline Normal or near normal & BNP/NT-proBNP plasma levels & Very elevated and rising \\
\hline No pericardial effusion; TAPSE $^{\star}>2.0 \mathrm{~cm}$ & Echocardiographic findings & Pericardial effusion; TAPSE $^{*}<1.5 \mathrm{~cm}$ \\
\hline Pra $<8 \mathrm{mmHg}$ and $\mathrm{Cl} \geqslant 2.5 \mathrm{~L} \cdot \mathrm{min}^{-1} \cdot \mathrm{m}^{-2}$ & Haemodynamics & Pra $>15 \mathrm{mmHg}$ or $\mathrm{Cl} \leqslant 2.0 \mathrm{~L} \cdot \mathrm{min}^{-1} \cdot \mathrm{m}^{-2}$ \\
\hline
\end{tabular}

WHO FC: World Health Organization functional class; 6MWT: 6-min walk test; BNP: brain natriuretic peptide; NT-proBNP: N-terminal pro-B-type natriuretic peptide; TAPSE: tricuspid annular plane systolic excursion; Pra: right atrial pressure; Cl: cardiac index. ${ }^{*}$ : depending on age; ": TAPSE and pericardial effusion have been selected as they can be measured in the majority of patients. Reproduced from [9] with permission from the publisher.

resistance [15] and changes in PVR [16]. Per cent predicted 6MWD offers no advantage over absolute distance [17].

Absolute distance walked after the commencement of therapy rather than change in $6 \mathrm{MWD}$ has been shown to be predictive of outcome [11, 15, 18]. This is reflected in treatment guidelines which state that $6 \mathrm{MWD}>500 \mathrm{~m}$ should be a goal of therapy, while a $6 \mathrm{MWD}<300 \mathrm{~m}$ should prompt intensification of treatment [9]. Systematic reviews of clinical trials in PAHspecific therapies have found that, while these treatment strategies have resulted in a significant reduction in all-cause mortality, this reduction bears no relationship with the change from baseline in 6MWD, and changes in 6MWD do not predict a survival benefit $[19,20]$. Although this may appear counterintuitive at first sight, it is apparent that prognosis does not depend on how much a patient has improved, rather how good their current status is. A patient with poor baseline 6MWD can improve by a considerable distance but still be severely compromised, whereas other less functionally impaired patients only need to improve by a short distance to achieve results which are associated with better cardiac function.

The precise definition of what constitutes a relevant change in 6MWD is complex, and it is essential to distinguish between changes that are clinically important or meaningful to the patient, and those that are trivial to the patient, despite being statistically significant in clinical trials. GILBERT et al. [21] calculated the smallest difference in 6MWD that signified a clinically important change (minimally important difference; MID) using data from patients in WHO FC II/III treated with sildenafil as part of the double blind, placebo-controlled SUPER (Sildenafil Use in Pulmonary Hypertension) study. Since there is no consensus regarding the best method for calculating MID, the authors used a number of statistical methods to calculate a MID of $40.7 \mathrm{~m}$, with a range of 18.7$74.15 \mathrm{~m}$ depending on which aspect of the SF-36 score was used. To the author's knowledge, no MID for 6MWD has been defined to predict a significant change in prognosis.

Unfortunately, 6MWD does not solely reflect cardiac function, as there can be many confounding factors such as motivation and mood, comorbidities (for example, connective tissue diseases, such as SSc [13]), and patient factors such as age, height and weight. In particular, muscular function and training have been shown to significantly affect 6MWD [22]. For example, in a study of patients with severe $\mathrm{PAH}$, exercise and respiratory training resulted in a significant increase in 6MWD (mean $111 \mathrm{~m}$, range 65-139 m) compared with untrained control subjects without any measurable effect on right ventricular function [23]. There is also evidence that the sensitivity of the $6 \mathrm{MWD}$ may decline in line with increasing baseline distance walked; treatments that improved haemodynamics and WHO FC have been found to have no significant effect on the 6MWD in patients with $\mathrm{PAH}$ with baseline 6MWD $>450 \mathrm{~m}$ [24]. It should also be noted that 6MWD has not been fully validated in patients with forms of $\mathrm{PAH}$ other than idiopathic PAH [9].

CPET offers a number of potential advantages over 6MWD in assessing a patient's exercise capacity. In patients with better baseline exercise capacity, for example, changes in 6MWD may be limited by a "ceiling effect" and so may not accurately reflect response to therapy. This is less of a problem using CPET as it is a maximal, symptom-limited test.

Guidelines recommend the use of peak oxygen consumption as a prognostic parameter, where values $>15 \mathrm{~mL} \cdot \mathrm{min}^{-1} \cdot \mathrm{kg}^{-1}$ are associated with better prognosis and values $<12 \mathrm{~mL} \cdot \mathrm{min}^{-1} \cdot \mathrm{kg}^{-1}$ with worse prognosis [9]. However, the need for a maximal effort in order to obtain the most useful diagnostic information can be problematic for some patients with severe disease or other cardiopulmonary comorbidities, and patient motivation may result in increased variability and decreased reproducibility of results. Effort-independent measures, such as the ventilation/carbon dioxide production slope, which can be derived even in the absence of a maximal effort, have been shown to be prognostic in patients with heart failure. This may also hold promise for $\mathrm{PAH}$, but needs further evaluation.

Despite recommendations, there is a lack of generally accepted standards for CPET in PAH in terms of standardisation of data acquisition and analysis. In addition, CPET requires specific exercise equipment and measurement systems, and needs to be performed and assessed by highly experienced technical and medical personnel, which makes it less convenient to perform than 6MWD. 


\section{Biomarkers}

The use of noninvasive biomarkers for the assessment of PAH and its progression is an attractive option. The most widely used and investigated in $\mathrm{PAH}$ is brain natriuretic peptide (BNP). BNP is secreted by ventricular myocytes and plays an important role in the regulation of blood pressure, blood volume and sodium balance. On secretion, the BNP precursor is split into the biologically active peptide and the more stable $\mathrm{N}$-terminal fragment (NT-proBNP) [25]. The production of $\mathrm{BNP} / \mathrm{NT}$-proBNP is augmented in response to factors that increase cardiac wall stress and, thus, cardiomyocyte stretch. Serum BNP/NT-proBNP levels have been shown to reflect right ventricular dysfunction severity in $\mathrm{PAH}$, correlating with mean $P$ pa, $P$ ra, total pulmonary resistance and right ventricle mass, and inversely correlating with cardiac output and ejection fraction [26, 27]. High plasma levels, and in particular a further increase in plasma BNP during follow-up, have a strong independent association with mortality [27, 28]. Guidelines recommend that $\mathrm{BNP} / \mathrm{NT}$-proBNP plasma levels are measured for initial risk stratification, and that they may be considered for monitoring the effects of treatment [9]. However, cut-off values for BNP/NT-proBNP levels have not been validated in outcome trials and, reflecting this, treatment guidelines refer only to "elevated" and "normal/near-normal" levels. Therefore, while a change in levels will give an indication of an improvement or decline in a patient's condition, absolute values of BNP/NTproBNP currently cannot be used as an indication of the degree of the associated improvement or decline.

\section{Echocardiography}

Noninvasive assessment of right heart function using echocardiography is an accepted and routine method for diagnosis and assessment of PAH. Numerous studies have demonstrated that echocardiographic parameters can be used as surrogate markers and are predictive of survival (table 2). One of the most frequently reported prognostic factors is the presence of pericardial effusion [3, 29-31]. Pericardial effusion in $\mathrm{PAH}$ is caused by impaired venous and lymphatic drainage of the myocardium linked to increased Pra and it is, therefore, a reflection of right ventricular (diastolic) dysfunction and right heart failure $[29,30]$. Another measure shown to have prognostic value in $\mathrm{PAH}$ is the tricuspid annular plane systolic excursion (TAPSE), a relatively simple measure of the longitudinal movement of the lateral tricuspid annulus towards the right ventricle apex. TAPSE has been shown to correlate with right ventricular ejection fraction $[32,33]$ and a TAPSE of $<1.8 \mathrm{~cm}$ is associated with greater right ventricle systolic dysfunction (lower cardiac index and right ventricle fractional

TABLE 2 Echocardiographic measures predictive of survival

Echocardiographic parameters

Worse prognosis

\begin{tabular}{lc} 
Tricuspid annular plane systolic excursion & $<15 \mathrm{~mm}$ \\
Right ventricular Doppler (Tei) index & $>0.88$ \\
Pericardial effusion & Present \\
Left ventricular eccentricity index at end-diastole & $>1.7$ \\
Right atrial volume & Increasing size \\
Right ventricular fractional area change & Decreasing \% \\
\hline
\end{tabular}

area change) and diastolic dysfunction (right atrial area index); it is also associated with decreased survival [34]. Pericardial effusion and TAPSE are recommended by treatment guidelines as a prognostic indicator in PAH for the assessment of disease severity and response to therapy, largely because both parameters are relatively easy to assess in the majority of patients [9]. However, other echocardiographic parameters of right heart function and structure have also been linked to prognosis in $\mathrm{PAH}$, including the myocardial performance or Tei index (calculated from the sum of the isovolumic contraction and isovolumic relaxation times divided by ejection time), which represents an estimate of global right ventricular performance [35]. The Tei index increases with increasing right ventricle dysfunction, and has been shown to be a prognostic indicator in $\mathrm{PAH}[36,37]$. Other reported prognostic parameters include the left ventricular eccentricity index [30], right ventricle dyssynchrony [38], right ventricle diameter and wall thickness [39], and right ventricular systolic to diastolic duration ratio [40]. Although the relative values of these measures remain to be fully established, it is likely that a comprehensive assessment of prognosis will require the evaluation of a number of different echocardiographic parameters. For example, in a study by GHIO et al. [39], a TAPSE of $<15 \mathrm{~mm}$ was associated with a significantly higher risk of mortality (20.8 per 100 person-yrs) compared with a TAPSE of $>15 \mathrm{~mm}$ (6.5 per 100 person-yrs). However, in patients with TAPSE $<15 \mathrm{~mm}$, a left ventricular eccentricity index at end-diastole $\geqslant 1.7$ was associated with the highest risk of mortality (51.7 per 100 person-yrs). Importantly, since TAPSE is a load-dependent measure, in patients with a TAPSE of $>15 \mathrm{~mm}$, the presence of severe tricuspid regurgitation markedly increased the risk of death (19.5 per 100 personyrs) compared with those with lesser tricuspid regurgitation (1.3 per 100 person-yrs) [39].

Although echocardiography is a well-established technique that is widely available, inexpensive and safe, and has proven value in the screening and management of patients with $\mathrm{PAH}$, it can be associated with a number of technical drawbacks and limitations around operator dependency and problems with obtaining suitable images [32]. In addition, Doppler-derived pressure estimation may be inaccurate in individual patients [41] and pressure may be consistently underestimated in the presence of severe tricuspid regurgitation [9]. Finally, although it is sometimes viewed as a key parameter, estimated pulmonary artery systolic pressure is not predictive of survival and should not be relied upon for patient assessment during followup visits.

\section{Haemodynamic measurements}

RHC is the gold standard for the diagnosis of PAH, with reassessment recommended 3-4 months after initiation of a new PAH treatment or in the case of clinical worsening [9]. Measurements made during RHC are also consistently predictive of survival. In patients included in the National Institutes of Health (NIH) primary pulmonary hypertension registry, who were studied before the advent of PAH-specific therapies, mean $P$ pa, mean Pra and cardiac index were all found to be predictive of survival [5], and mean $P$ pa and mean $P$ ra at baseline were prognostic factors for patients treated with epoprostenol [11]. Furthermore, reduction in total pulmonary resistance of at least 30\% [10] and improvements in cardiac 
index and mean $P$ pa [42] are associated with improved survival following a period of epoprostenol treatment. More recently, data from the French network on pulmonary hypertension showed that baseline Pra, cardiac index and cardiac output were also significantly associated with survival in the modern treatment era [4]. Data from the NIH registry were used to devise a formula to estimate survival in patients with $\mathrm{PAH}$ based on haemodynamic parameters (mean Ppa, mean Pra and cardiac index) [5]. This formula has been widely used in clinical trials without a control arm, where the observed survival rates following treatment with a study drug are compared with survival rates predicted by the $\mathrm{NIH}$ equation to suggest improvements in survival. However, the data behind the NIH equation are now more than two decades old and, as previously mentioned, come from patients managed before the availability of PAH-specific therapy; the relevance of the equation in the modern treatment era is therefore uncertain. THENAPPAN et al. [43] recently demonstrated this, using data from a contemporary patient cohort and found that survival was better in the modern day cohort compared with that predicted by the NIH registry equation, and concluded that the NIH equation underestimated survival in the current treatment era. A new survival prediction equation was developed using the same variables as the NIH equation, and predicted survival using this new equation was shown to be comparable to actual observed survival in a number of other published contemporary patient cohorts [43].

Although considered to be the "gold standard" for the diagnosis of PAH, there are some disadvantages to using RHC as a followup procedure. It is invasive and needs to be performed in centres with the appropriate expertise. Therefore, it is not always practical as a regular follow-up procedure for some patients. In addition, in the author's view, if results from all other parameters (e.g. echocardiography, exercise testing, serum biomarkers) are in agreement, haemodynamic measures may not add any further information in guiding treatment and, therefore, RHC may not always be required for monitoring purposes.

What has been described thus far are a number of tests and parameters that are linked directly to right heart structure and function and that have been shown to have prognostic value in PAH. It is clear that these measures should not be used in isolation, and that it is important to combine results from a number of parameters to obtain a true reflection of prognosis in PAH. However, the most appropriate combination of factors and the optimal levels or cut-off values which give the most accurate prognosis in an individual patient are currently undefined.

\section{USING DATA FROM LARGE REGISTRIES}

In addition to the individual studies investigating potential prognostic factors discussed above, there are now an abundance of real-world data available from PAH registries. The data from such registries are useful to describe and compare patient characteristics, practice patterns and outcomes in different patient populations over time. Observations from registries can also be used to raise questions and hypotheses which can be tested in future trials, and to test the validity of prognostic indicators. Recent data from the French network on pulmonary hypertension and the US REVEAL registry (Registry to Evaluate Early and Long-term PAH Disease Management) confirmed the prognostic value of simple assessments such as WHO FC and 6MWD, and the importance of Pra and cardiac index [3, 4]. Some of these prognostic indicators have been used to draw up predictive equations as previously discussed [4, 5, 43], and it is tempting to suggest that the use of such equations may allow the individualisation and optimisation of therapeutic strategies, however, this approach has never been systematically evaluated.

\section{FUTURE CONTROVERSIES RELATING TO PATIENT PROGNOSIS}

Although there has been considerable improvement in our understanding of $\mathrm{PAH}$ and its management over the past decade, there is still much that requires further investigation. In particular, despite its importance, the right ventricle has been less well studied, as have the processes underlying pulmonary vascular remodelling and strategies to modify it. There is still much to learn about the assessment of right heart function, and we have not yet identified the ideal surrogate markers in PAH. Catheterisation remains the gold standard, but is invasive. An underexplored area of study is whether exercise haemodynamics can add value in assessing a patient's prognosis or response to therapy, but this clearly adds complexity to the procedure. Evidence is accumulating for an additive role of measurements on incremental exercise in predicting prognosis. Combining peak oxygen uptake and peak systolic blood pressure is a more robust method of predicting survival than using the two measurements independently [44] and, more recently, it has been suggested that the ventilatory equivalent for carbon dioxide at the anaerobic threshold might add prognostic value to the 6MWD (R. Naeije, Erasme University Hospital, Brussels, Belgium; personal communication). This latter observation suggests that measurements made at submaximal exercise may be sufficient for the assessment of cardiopulmonary function $[45,46]$.

Current guidelines recommend the use of a combination of parameters with established prognostic indicators to assess disease severity and to guide treatment. However, what exactly defines "good prognosis" for these markers is not clear cut, and limits may not be the same for all patients; the prognosis for a 30 -yr-old with a 6MWD of $400 \mathrm{~m}$ is unlikely to be the same as for a 70 -yr-old with the same walk distance. Although prediction equations have been drawn up and appear to perform well, such equations are developed using data from limited cohorts of patients and, therefore, may not be generalisable to the wider patient population [43]. Whether it might be possible to develop a prognostic equation sophisticated enough to be applied to an individual patient remains to be seen.

\section{CONCLUSION}

Many measures are available for the evaluation of severity in $\mathrm{PAH}$ and form the basis of recommendations for the assessment of disease severity and response to therapy. When examined, these measures inform us more about the function of the right ventricle rather than the status of the pulmonary vasculature. This is no coincidence, as cardiac status underpins symptoms and prognosis in PAH. However, individual parameters considered on their own may be misleading and need to be considered in the context of other demographic factors, such as age, as well as other measurements. Multifactorial equations may overcome these issues, but are complex by necessity, and it is not clear if they can provide a real advantage when managing individuals over the clinician who can integrate results in his or her mind to best treat the patient. The role of multifactorial 
equations may be confined to comparative audit and research. With plenty of tools at our disposal, there is still room to refine current measurements and to understand them better. There is also an increasing need to evaluate second- and third-line novel agents and we need to understand which end-points are going to help us take drugs through to the final stages of development.

\section{STATEMENT OF INTEREST}

L.S. Howard has received honoraria for speaking and acting on advisory boards from Actelion, Pfizer, GSK and Bayer. He has received research grants from Pfizer and Bayer, and funds for a member of staff from Actelion. Travel and conference fees have been provided by Actelion, GSK and Pfizer.

\section{ACKNOWLEDGEMENTS}

I received editorial assistance from L. Thomas (Elements Communications Ltd, Westerham, UK), supported by Actelion Pharmaceuticals Ltd. (Allschwil, Switzerland).

\section{REFERENCES}

1 Humbert M, Sitbon O, Simonneau G. Treatment of pulmonary arterial hypertension. N Engl J Med 2004; 351: 1425-1436.

2 Chin KM, Kim NH, Rubin LJ. The right ventricle in pulmonary hypertension. Coron Artery Dis 2005; 16: 13-18.

3 Benza RL, Miller DP, Gomberg-Maitland M, et al. Predicting survival in pulmonary arterial hypertension: insights from the Registry to Evaluate Early and Long-Term Pulmonary Arterial Hypertension Disease Management (REVEAL). Circulation 2010; 122: $164-172$.

4 Humbert M, Sitbon O, Chaouat A, et al. Survival in patients with idiopathic, familial, and anorexigen-associated pulmonary arterial hypertension in the modern management era. Circulation 2010; 122: $156-163$

5 D'Alonzo GE, Barst RJ, Ayres SM, et al. Survival in patients with primary pulmonary hypertension. Results from a national prospective registry. Ann Intern Med 1991; 115: 343-349.

6 Galiè N, Rubin LJ, Hoeper M, et al. Treatment of patients with mildly symptomatic pulmonary arterial hypertension with bosen$\tan$ (EARLY study): a double-blind, randomised controlled trial. Lancet 2008; 371: 2093-2100.

7 Bogaard HJ, Abe K, Vonk Noordegraaf A, et al. The right ventricle under pressure: cellular and molecular mechanisms of right-heart failure in pulmonary hypertension. Chest 2009; 135: 794-804.

8 Champion HC, Michelakis ED, Hassoun PM. Comprehensive invasive and noninvasive approach to the right ventricle-pulmonary circulation unit: state of the art and clinical and research implications. Circulation 2009; 120: 992-1007.

9 Galiè N, Hoeper MM, Humbert M, et al. Guidelines for the diagnosis and treatment of pulmonary hypertension: the Task Force for the Diagnosis and Treatment of Pulmonary Hypertension of the European Society of Cardiology (ESC) and the European Respiratory Society (ERS), endorsed by the International Society of Heart and Lung Transplantation (ISHLT). Eur Heart J 2009; 30: 2493 2537

10 Le RJ, Fenstad ER, Maradit-Kremers H, et al. Syncope in adults with pulmonary arterial hypertension. J Am Coll Cardiol 2011; 58: 863-867.

11 Sitbon $\mathrm{O}$, Humbert $\mathrm{M}$, Nunes $\mathrm{H}$, et al. Long-term intravenous epoprostenol infusion in primary pulmonary hypertension: prognostic factors and survival. J Am Coll Cardiol 2002; 40: 780-788.

12 Barst RJ, Gibbs JS, Ghofrani HA, et al. Updated evidence-based treatment algorithm in pulmonary arterial hypertension. J Am Coll Cardiol 2009; 54: Suppl. 1, S78-S84.

13 Distler O, Behrens F, Pittrow D, et al. Defining appropriate outcome measures in pulmonary arterial hypertension related to systemic sclerosis: a Delphi consensus study with cluster analysis Arthritis Rheum 2008; 59: 867-875.

14 Hachulla E, Launay D, Yaici A, et al. Pulmonary arterial hypertension associated with systemic sclerosis in patients with functional class II dyspnoea: mild symptoms but severe outcome. Rheumatology (Oxford) 2010; 49: 940-944.

15 Miyamoto S, Nagaya N, Satoh T, et al. Clinical correlates and prognostic significance of six-minute walk test in patients with primary pulmonary hypertension. Comparison with cardiopulmonary exercise testing. Am J Respir Crit Care Med 2000; 161: 487-492.

16 FDA Advisory Committee Briefing Document Cardiovascular and Renal Drugs Advisory Committee. Use of $\triangle$ PVRI for dosing recommendations of adult-approved drugs in pediatric $\mathrm{PAH}$ patients. www.fda.gov/downloads/AdvisoryCommittees/CommitteesMeet ingMaterials/Drugs/CardiovascularandRenalDrugsAdvisoryCom mittee/UCM220250.pdf Date last updated: July 29, 2010. Date last accessed: July 21, 2011.

17 Lee WT, Peacock AJ, Johnson MK. The role of per cent predicted 6min walk distance in pulmonary arterial hypertension. Eur Respir J 2010; 36: 1294-1301.

18 Paciocco G, Martinez FJ, Bossone E, et al. Oxygen desaturation on the six-minute walk test and mortality in untreated primary pulmonary hypertension. Eur Respir J 2001; 17: 647-652.

19 Macchia A, Marchioli R, Marfisi R, et al. A meta-analysis of trials of pulmonary hypertension: a clinical condition looking for drugs and research methodology. Am Heart J 2007; 153: 1037-1047.

20 Macchia A, Marchioli R, Tognoni G, et al. Systematic review of trials using vasodilators in pulmonary arterial hypertension: why a new approach is needed. Am Heart J 2010; 159: 245-257.

21 Gilbert C, Brown MC, Cappelleri JC, et al. Estimating a minimally important difference in pulmonary arterial hypertension following treatment with sildenafil. Chest 2009; 135: 137-142.

22 Fox BD, Kassirer M, Weiss I, et al. Ambulatory rehabilitation improves exercise capacity in patients with pulmonary hypertension. J Card Fail 2011; 17: 196-200.

23 Mereles D, Ehlken N, Kreuscher S, et al. Exercise and respiratory training improve exercise capacity and quality of life in patients with severe chronic pulmonary hypertension. Circulation 2006; 114: 1482-1489.

24 Degano B, Sitbon O, Savale L, et al. Characterization of pulmonary arterial hypertension patients walking more than $450 \mathrm{~m}$ in $6 \mathrm{~min}$ at diagnosis. Chest 2010; 137: 1297-1303.

25 Yap LB. B-type natriuretic peptide and the right heart. Heart Fail Rev 2004; 9: 99-105.

26 Nagaya N, Nishikimi T, Okano Y, et al. Plasma brain natriuretic peptide levels increase in proportion to the extent of right ventricular dysfunction in pulmonary hypertension. J Am Coll Cardiol 1998; 31: 202-208.

27 Fijalkowska A, Kurzyna M, Torbicki A, et al. Serum N-terminal brain natriuretic peptide as a prognostic parameter in patients with pulmonary hypertension. Chest 2006; 129: 1312-1321.

28 Nagaya N, Nishikimi T, Uematsu M, et al. Plasma brain natriuretic peptide as a prognostic indicator in patients with primary pulmonary hypertension. Circulation 2000; 102: 865-870.

29 Raymond RJ, Hinderliter AL, Willis PW, et al. Echocardiographic predictors of adverse outcomes in primary pulmonary hypertension. J Am Coll Cardiol 2002; 39: 1214-1219.

30 Hinderliter AL, Willis PW 4th, Barst RJ, et al. Effects of long-term infusion of prostacyclin (epoprostenol) on echocardiographic measures of right ventricular structure and function in primary pulmonary hypertension. Primary Pulmonary Hypertension Study Group. Circulation 1997; 95: 1479-1486.

31 Zhang R, Dai LZ, Xie WP, et al. Survival of Chinese patients with pulmonary arterial hypertension in the modern management era. Chest 2011; 140: 301-309.

32 Badano LP, Ginghina C, Easaw J, et al. Right ventricle in pulmonary arterial hypertension: haemodynamics, structural 
changes, imaging, and proposal of a study protocol aimed to assess remodelling and treatment effects. Eur J Echocardiogr 2010; 11: 27-37.

33 Miller D, Farah MG, Liner A, et al. The relation between quantitative right ventricular ejection fraction and indices of tricuspid annular motion and myocardial performance. J Am Soc Echocardiogr 2004; 17: 443-447.

34 Forfia PR, Fisher MR, Mathai SC, et al. Tricuspid annular displacement predicts survival in pulmonary hypertension. Am J Resp Crit Care Med 2006; 174: 1034-1041.

35 Tei C, Dujardin KS, Hodge DO, et al. Doppler echocardiographic index for assessment of global right ventricular function. J Am Soc Echocardiogr 1996; 9: 838-847.

36 Yeo TC, Dujardin KS, Tei C, et al. Value of a Doppler-derived index combining systolic and diastolic time intervals in predicting outcome in primary pulmonary hypertension. Am J Cardiol 1998; 81: 1157-1161.

37 Grapsa I, Pavlopoulos H, Dawson D, et al. Retrospective study of pulmonary hypertensive patients: is right ventricular myocardial performance index a vital prognostic factor? Hellenic J Cardiol 2007; 48: 152-160.

38 Lopez-Candales A, Rajagopalan N, Saxena N, et al. Right ventricular systolic function is not the sole determinant of tricuspid annular motion. Am J Cardiol 2006; 98: 973-977.

39 Ghio S, Klersy C, Magrini G, et al. Prognostic relevance of the echocardiographic assessment of right ventricular function in patients with idiopathic pulmonary arterial hypertension. Int $J$ Cardiol 2010; 140: 272-278.

40 Alkon J, Humpl T, Manlhiot C, et al. Usefulness of the right ventricular systolic to diastolic duration ratio to predict functional capacity and survival in children with pulmonary arterial hypertension. Am J Cardiol 2010; 106: 430-436.

41 Mukerjee D, St George D, Knight C, et al. Echocardiography and pulmonary function as screening tests for pulmonary arterial hypertension in systemic sclerosis. Rheumatology (Oxford) 2004; 43: 461-466.

42 McLaughlin VV, Shillington A, Rich S. Survival in primary pulmonary hypertension: the impact of epoprostenol therapy. Circulation 2002; 106: 1477-1482.

43 Thenappan T, Shah SJ, Rich S, et al. Survival in pulmonary arterial hypertension: a reappraisal of the NIH risk stratification equation. Eur Respir J 2010; 35: 1079-1087.

44 Wensel R, Opitz CF, Anker SD, et al. Assessment of survival in patients with primary pulmonary hypertension: importance of cardiopulmonary exercise testing. Circulation 2002; 106: 319-324.

45 Woods PR, Frantz RP, Taylor BJ, et al. The usefulness of submaximal exercise gas exchange to define pulmonary arterial hypertension. J Heart Lung Transplant 2011; [Epub ahead of print DOI: 10.1016/j.healun.2011.03.021].

46 Lee WT, Brown A, Peacock AJ, et al. Use of non-invasive haemodynamic measurements to detect treatment response in precapillary pulmonary hypertension. Thorax 2011; 66: 810-814. 